\title{
Evaluation of a Pharmacist-Managed Nonsteroidal Anti-Inflammatory Drugs Deprescribing Program in an Integrated Health Care System
}

\author{
Rezoana Rashid, PharmD, BCPS; Christopher Chang, PharmD, BCGP, PMP; Fang Niu, MS; \\ Lynn Deguzman, PharmD, BCGP; Maisha Draves, MD, MPH; Sameer Awsare, MD, FACP; \\ and Rita Hui, PharmD, MS
}

\begin{abstract}
BACKGROUND: Nonsteroidal anti-inflammatory drugs (NSAIDs) are commonly used to treat symptoms of chronic inflammatory diseases such as osteoarthritis and rheumatoid arthritis; however, they are also associated with various adverse effects, including gastrointestinal (GI) bleeding and renal harm. As patients get older, some medications may no longer be beneficial or may even cause harm. Deprescribing is defined as the planned and supervised process of dose reduction or discontinuation of medications. While there are studies showing that deprescribing strategies with several classes of medications positively affects outcomes in elderly patients, there is a lack of strong evidence and guidance to deprescribe NSAIDs.
\end{abstract}

OBJECTIVE: To evaluate the effectiveness, safety, and economic impact of pharmacists deprescribing NSAIDs under the guidance of a standardized deprescribing program compared with usual care within an integrated health care system.

METHODS: This retrospective, propensity score-matched cohort study included patients aged $\geq 65$ years who were eligible for the NSAID deprescribing program from July 2016 to June 2018. Those patients in the deprescribing group were assessed by pharmacists and had their medications deprescribed. Patients who were eligible for the deprescribing program but did not receive any interventions were matched to the deprescribed group using propensity score matching at a $4: 1$ ratio and became the usual care group. Patients were followed for 6 months, until end of membership, or until death, whichever occurred first. The effectiveness and safety outcomes included rates of 3 adverse events: GI bleeds, acute kidney injuries (AKI), and exacerbation of pain triggering a hospitalization or emergency room visit. The economic outcome was the change in monthly NSAID cost. Descriptive statistics, t-tests, chi-square tests, and conditional logistic regression models were used for analysis.

RESULTS: There were 431 patients in the deprescribed group and 1,724 patients in the usual care group, with similar baseline characteristics after propensity score matching. The adjusted results showed no significant difference between the deprescribed and usual care groups for GI bleed events $(\mathrm{OR}=0.65,95 \% \mathrm{Cl}=0.36-1.16, P=0.15)$ and $\mathrm{AKI}(\mathrm{OR}=0.53$, $95 \% \mathrm{Cl}=0.24-1.16, P=0.11)$. The deprescribed group experienced a significant 2-fold decrease in the odds of exacerbation of pain versus the deprescribed group $(0 \mathrm{R}=0.50,95 \% \mathrm{Cl}=0.33-0.77, P<0.01)$. Finally, there was no significant difference in the change in monthly NSAIDs costs between the 2 groups (median change, IQR: $-\$ 0.29$, $-\$ 2.37$ to $-\$ 0.11$ for deprescribed group; $-\$ 0.23,-2.59$ to 0.00 for usual care group, $P=0.054$ ). CONCLUSIONS: Although this study did not find any difference in the rate of GI bleed or AKI, we found a significant decrease in the rate of exacerbation of pain in the deprescribed group versus the usual care group. This result suggests that deprescribing NSAIDs did not cause harm during the 6-month follow-up. Further long-term studies are necessary to validate these outcomes.

J Manag Care Spec Pharm. 2020;26(7):918-24

Copyright $\odot 2020$, Academy of Managed Care Pharmacy. All rights reserved.

\section{What is already known about this subject}

Elderly patients are at risk of polypharmacy as they develop chronic conditions.

Chronic use of nonsteroidal anti-inflammatory drugs (NSAIDs) may lead to harmful adverse effects such as gastrointestinal (GI) bleed events and acute kidney injuries (AKI), especially in elderly patients with risk factors

There is currently a lack of quality evidence and guidance in deprescribing NSAIDs in elderly patients.

\section{What this study adds}

The outcomes of a pharmacist-managed NSAID deprescribing program aimed to reduce chronic NSAID use in elderly patients with risk factors was compared with a group of propensity scorematched usual care patients, who did not get the intervention. There was no difference in the odds of patients having a GI bleed or AKI among the 2 groups.

There was a statistically significant 2-fold decrease in the odds of patients experiencing exacerbation of pain requiring acute care in the pharmacist-managed deprescribed group versus the usual care group.

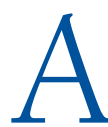
ccording to the U.S. Census Bureau, the geriatric population, which comprises individuals aged 65 years and above, will double from 40 million to 80 million in the next few decades. ${ }^{1}$ Elderly patients are often diagnosed with multiple chronic diseases that require medication therapy to control symptoms and improve clinical outcomes. Consequently, these patients are often burdened by polypharmacy-the concurrent use of multiple medications-which can place patients at increased risk of mortality, falls, adverse drug reactions, increased hospital length of stay, and readmissions. Elderly patients are also at greater risk for decreased renal and hepatic function and lower lean body mass, all of which may potentiate the risks of polypharmacy. ${ }^{2}$

Deprescribing is defined as a planned and supervised process of dose reduction or discontinuation of medications that may lead to harm or are no longer beneficial for the patient. ${ }^{3}$ Deprescribing can be used as a strategy to reduce medication overuse from polypharmacy in the geriatric population. Early 
studies with deprescribing strategies for antidiabetics, benzodiazepines, antipsychotics, and proton-pump inhibitors have shown improved outcomes in elderly patients. ${ }^{4-7}$ Recently, a study evaluated a pharmacist-managed antidiabetic deprescribing program at Kaiser Permanente Northern California (KPNC). Key effectiveness and safety findings included a decrease in the rate of hypoglycemia and a nonclinically significant increase in hemoglobin Alc. The study confirmed the safety endpoints by observing no significant differences in the rate of hyperglycemia events or proportion of patients at goal $(\mathrm{Alc}<7 \%)$ between the deprescribed and usual care groups. ${ }^{5}$

Nonsteroidal anti-inflammatory drugs (NSAIDs) are commonly used to treat symptoms of chronic inflammatory diseases, such as osteoarthritis and rheumatoid arthritis. However, they are associated with various adverse effects, including gastrointestinal (GI) bleed events, cardiovascular events, and acute kidney injuries (AKI), that can cause hospitalizations and/or death. ${ }^{8,9}$ Data from structured interviews concluded that up to 33\% of patients aged 65 years and older were chronic users of NSAIDs. ${ }^{10}$ There is currently a lack of high-quality evidence and guidance in deprescribing NSAIDs in elderly patients. ${ }^{11}$

Under a partnership between the KPNC Clinical Pharmacy Services and The Permanente Medical Group in 2016, a collaborative physician-pharmacist deprescribing program was designed to reduce the use of chronic NSAIDs in geriatric patients with risk factors. The objective of this study was to evaluate effectiveness, safety, and economic outcomes of a pharmacist-managed deprescribing of NSAIDs within an integrated health care system.

\section{Methods}

\section{Setting}

KPNC is a large, integrated health care system that serves over 4 million members. KPNC has an electronic medical record (EMR) system that allows access to various clinical encounters, hospitalizations, and medication dispenses associated with the members. This retrospective, propensity score-matched cohort study was approved by the Institutional Review Board at KPNC. Informed consent was waived because of the retrospective nature of the study.

\section{Deprescribing Program Workflow}

Under a collaborative drug therapy management protocol, such as the deprescribing program, pharmacists have the authority under the scope of California law to initiate, adjust, or stop medications directly with patients. Clinical pharmacists at KPNC who work in the deprescribing program are residency-trained and/or hold board certification in a relevant area of pharmacy practice. EMRs are screened quarterly to identify eligible patients based on an internally created electronic algorithm. Once eligible patients are identified, clinical pharmacists obtain protocol authorization via the EMR from the patient's primary care provider to review for NSAID deprescribing interventions. After receiving approval, clinical pharmacists review each patient's medical chart to determine if the patient is a good candidate for deprescribing interventions. If so, the pharmacist contacts the patient via telephone to discuss an appropriate deprescribing intervention based on the patient's drug regimen, preference of interventions, and lifestyle. Typically, a dose reduction by $25 \%-50 \%$ or discontinuation of the NSAID is initially recommended. The patient-pharmacist interactions and shared decisions are documented in the patient's EMR. Finally, a follow-up is typically scheduled within 2 to 4 weeks, but the clinical pharmacists make themselves accessible via secure online messaging through Kaiser Permanente's member website (kp.org) or via telephone between visits. Patients would be monitored for pain control at scheduled follow-ups and would be assessed for the effectiveness of the deprescribing plan. If clinically necessary at follow-up, the pharmacist may retitrate the NSAID to the lowest effective dose. ${ }^{5}$

\section{Study Design and Populations}

To be eligible for the NSAID deprescribing program at KPNC, patients had to be aged at least 65 years and had to have received at least 270 days supply of NSAIDs, which included oxicam derivatives, acetic acid derivatives, propionic acid derivatives, salicylates, and cyclooxygenase-2 inhibitors, within a year before the program eligibility date. Patients also had to have at least 1 of the following risk factors that potentially could be exacerbated by NSAID use within the past 24 months to ensure that the risk factors were recent or current. These factors included diagnoses from the EMR for GI bleed or disorders (ulcer and severe esophagitis) and cardiovascular disease (myocardial infarction, stroke, transient ischemic attack, atherosclerotic cardiovascular disease, or heart failure) or end-stage renal disease using International Classification of Diseases, Ninth/Tenth Revision, Clinical Modification (ICD9/10-CM) codes or Disease-Related Group (DRG) codes (full list available in the Appendix, available in online article). Finally, usage of an anticoagulant or prednisone $>10 \mathrm{mg}$ per day or an equivalent systemic corticosteroid measured over the previous 12 months was also considered a risk factor.

The study cohort included patients who were eligible for the deprescribing program during the identification period from July 1, 2016, to June 30, 2018. The pharmacist-managed deprescribed group included patients who were contacted and had interventions done by a pharmacist for enrollment in the deprescribing program during the cohort identification period. The usual care group included patients who were eligible for the program during the cohort identification period but did not have their medical records reviewed nor had any interventions done by clinical pharmacists because of resource limitations. The index date for the deprescribed group was the initial pharmacist intervention date during the cohort identification 


\section{FIGURE 1 Patient Selection Flowchart}

Eligible Patients $(\mathrm{N}=2,887)$

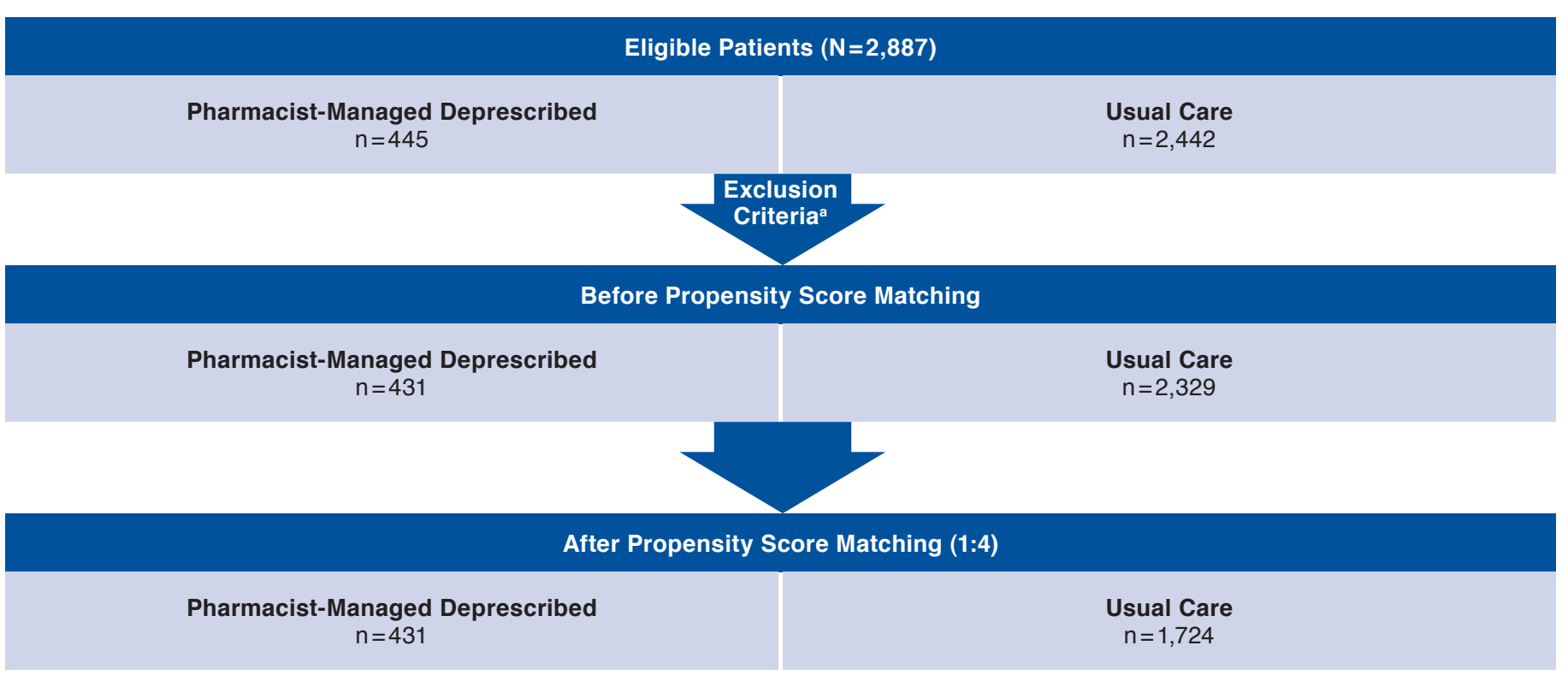

aExclusion Criteria: (1) no previous 6-month membership; (2) index date outside of inclusion period: pharmacist-managed: $n=4$, usual care: $n=72 ;$ and (3) no evidence of NSAID use within 90 days of index date: pharmacist-managed: $n=14$, usual care: $n=106$.

NSAID = nonsteroidal anti-inflammatory drug.

period. The index date for the usual care group was the first physician office visit after program eligibility identification. Patients were excluded if they were not members of KPNC for the 6 months before their index dates, had index dates outside of the inclusion period, and/or did not have evidence of NSAID use within 90 days before the index date.

A propensity score was calculated using various pretreatment characteristics as covariables in a logistic regression model to determine the likelihood that a patient would receive a clinical pharmacist intervention. These covariables included age at the time of index date; sex; race; Charlson Comorbidity Index (CCI) score ${ }^{12}$; proportion of patients with previous hospitalized GI bleed events 6 months before the index date; NSAID classes (oxicam derivatives, acetic acid derivatives, propionic acid derivatives, salicylates, and cyclooxygenase-2 inhibitors) received; and 8 pain-related diagnoses (general pain, headache, ankylosing spondylitis, gout, osteoarthritis, rheumatoid arthritis, joint pain, and muscle spasm) within 6 months before the index date that are associated with the use of NSAIDs. The usual care group patients were matched to the deprescribing group at a 4:1 ratio by selecting the closest propensity score without replacement. All patients were followed for 6 months or until end of membership or death, whichever occurred first.

\section{Effectiveness Outcomes}

To evaluate the effectiveness of the pharmacist-managed NSAID deprescribing program, this study examined the rate of new onset of GI bleed events and AKI, which was defined by any emergency room (ER) visit or hospitalization with a primary diagnosis of GI bleed or AKI using ICD-9/10-CM or DRG codes (Appendix).

\section{Safety Outcome}

The rate of ER visits or hospitalization because of exacerbation of pain or preexisting pain was used as a proxy for safety outcome. More specifically, safety outcomes evaluated the proportion of patients diagnosed within 6 months before the index date with at least 1 of the 8 pain-related diagnoses and then presented to the ER or hospitalized primarily because of an exacerbation of the same pain. The 8 pain diagnoses were defined using ICD-9/10-CM codes for general pain, headache, ankylosing spondylitis, gout, osteoarthritis, rheumatoid arthritis, joint pain, or muscle spasm (Appendix).

\section{Economic Outcome}

The economic outcome was the change in cost of NSAID prescriptions per member per month (PMPM). The change in cost of NSAIDs was calculated by subtracting the mean monthly NSAID drug cost within the previous 6 months from the mean monthly drug costs during the follow-up period for each patient. 


\section{TABLE 1 Baseline Demographics Before and After Propensity Score Matching}

\begin{tabular}{|c|c|c|c|c|c|}
\hline \multirow[b]{2}{*}{ Baseline Characteristic } & \multirow[b]{2}{*}{$\begin{array}{c}\text { Deprescribed } \\
n=431\end{array}$} & \multicolumn{2}{|c|}{$\begin{array}{c}\text { Before Propensity } \\
\text { Score Matching }\end{array}$} & \multicolumn{2}{|c|}{$\begin{array}{l}\text { After Propensity } \\
\text { Score Matching }\end{array}$} \\
\hline & & $\begin{array}{c}\text { Usual Care } \\
n=2,329\end{array}$ & $P$ Value ${ }^{a}$ & $\begin{array}{c}\text { Usual Care } \\
\mathrm{n}=1,724\end{array}$ & $P$ Value ${ }^{a}$ \\
\hline Age, mean $\pm S D^{b}$ & $76.1 \pm 6.7$ & $75.0 \pm 7.0$ & $<0.01$ & $75.8 \pm 7.1$ & 0.23 \\
\hline Female, $\mathrm{n}(\%)^{\mathrm{c}}$ & $232(53.8)$ & $1,353(58.1)$ & 0.09 & $950(55.1)$ & 0.63 \\
\hline Caucasian, $\mathrm{n}(\%)^{\mathrm{c}}$ & $355(82.4)$ & $1,843(79.1)$ & 0.13 & $1,410 \quad(81.8)$ & 0.78 \\
\hline Charlson Comorbidity Index score, median (IQR)b & $1 \quad(1-2)$ & $1 \quad(1-3)$ & 0.13 & $1 \quad(1-2)$ & 0.55 \\
\hline Patients with previous hospitalized GI bleed events, n (\%)c & $37 \quad(8.6)$ & $279(12.0)$ & 0.04 & $152 \quad(8.8)$ & 0.88 \\
\hline \multicolumn{6}{|l|}{ NSAID class, $\mathbf{n}(\%)^{c}$} \\
\hline Oxicam derivatives & $211 \quad(48.9)$ & $938(40.3)$ & \multirow{4}{*}{$<0.01$} & $796(46.2)$ & \multirow{4}{*}{0.84} \\
\hline Acetic acid derivatives & $122(28.3)$ & $677(29.1)$ & & $496(28.8)$ & \\
\hline Propionic acid derivatives & $76 \quad(17.6)$ & $517(22.2)$ & & $329 \quad(19.1)$ & \\
\hline Other & $22 \quad(5.1)$ & $197 \quad(8.5)$ & & $103 \quad(5.9)$ & \\
\hline \multicolumn{6}{|l|}{ Pain-related diagnoses associated with use of NSAIDs, $\mathbf{n}(\%)^{c}$} \\
\hline General pain & $185(42.9)$ & $1,113(47.8)$ & 0.06 & $779 \quad(45.2)$ & 0.40 \\
\hline Headache & $45(10.4)$ & $331 \quad(14.2)$ & 0.04 & $181(10.5)$ & 0.97 \\
\hline Ankylosing spondylitis & $81(18.8)$ & $573(24.6)$ & $<0.01$ & $349(20.2)$ & 0.50 \\
\hline Gout & $25 \quad(5.8)$ & $151 \quad(6.5)$ & 0.60 & $101 \quad(5.9)$ & 0.96 \\
\hline Osteoarthritis & $164(38.1)$ & $1,102 \quad(47.3)$ & $<0.01$ & $705 \quad(40.9)$ & 0.28 \\
\hline Rheumatoid arthritis & $13 \quad(3.0)$ & $131 \quad(5.6)$ & 0.03 & $63 \quad(3.7)$ & 0.52 \\
\hline Joint pain & $227(52.7)$ & $1,448 \quad(62.2)$ & $<0.01$ & $953(55.3)$ & 0.33 \\
\hline Muscle spasm & $17 \quad(3.9)$ & $104 \quad(4.5)$ & 0.63 & $70 \quad(4.1)$ & 0.91 \\
\hline
\end{tabular}

\section{Statistical Analysis}

Approximately 272 patients in the pharmacist-managed group and 1,088 patients in the usual care group were required to achieve $80 \%$ power with a type I error of $5 \%$ in order to detect a decrease from $11 \%$ to $5 \%$ in the proportion of patients who experience GI bleeds. ${ }^{9}$ The baseline characteristics of the deprescribed and usual care groups were compared before and after propensity score matching using descriptive analyses. Differences between groups were assessed using Student's t-tests or Wilcoxon rank-sum tests for the continuous variables and Pearson chi-square tests for the categorical variables. Conditional logistic regressions were conducted to determine the risk of GI bleed events, AKI, and exacerbation of pain. For effectiveness and safety outcomes, the common independent variables used in the conditional logistic regression model included age, sex, CCI score, Caucasian, NSAID classes, and 8 pain diagnoses. Specifically, for the risk of GI bleed, we also adjusted for previous hospitalized GI bleed events, whereas for risk of AKI and exacerbation of pain, we also adjusted for renal disease. $P$ values $<0.05$ were considered statistically significant. The SAS statistical package version 9.4 (SAS Institute, Cary, NC) was used to perform all analyses.

\section{Results}

\section{Patients}

During the cohort identification period, 2,887 patients were eligible for the deprescribing program. After applying the inclusion and exclusion criteria, there were 431 patients in the deprescribed group and 2,329 patients in the usual care group (Figure 1). Before propensity score matching, several baseline variables were significantly different between the 2 groups (Table 1). After performing propensity score matching, 1,724 patients from the 2,329 patients in the usual care group were selected to achieve a ratio of $4: 1$ to the deprescribing group (Figure 1). There were no statistical differences between the 2 matched groups in terms of age, sex, race, CCI score, previous hospitalized GI bleed events, NSAID classes, and painrelated diagnoses associated with the use of NSAIDs (Table 1). The deprescribed group and usual care group had a mean age (standard deviation [SD]) of $76.1( \pm 6.7)$ and $75.8( \pm 7.1)$ years, respectively $(P=0.23)$. In both groups, over $50 \%$ of the patients were female; over $80 \%$ were Caucasian; and approximately $50 \%$ used oxicam derivatives (Table 1). 


\section{TABLE 2 Unadjusted Outcomes}

\begin{tabular}{|c|c|c|c|}
\hline & Deprescribed, $n=431$ & Usual Care, $\mathbf{n}=1,724$ & $P$ Value \\
\hline \multicolumn{4}{|l|}{ Effectiveness } \\
\hline Composite GI bleed events, n (\%)a,b & $(3.9)$ & $(6.6)$ & 0.04 \\
\hline ER visits & $(2.3)$ & $(3.1)$ & 0.37 \\
\hline Hospitalization & $(2.6)$ & $(5.1)$ & 0.03 \\
\hline Composite AKI, n (\%)a,b & $(2.3)$ & (3.9) & 0.11 \\
\hline ER visits & $(2.1)$ & $(3.1)$ & 0.25 \\
\hline Hospitalization & $(2.3)$ & $66 \quad(3.8)$ & 0.13 \\
\hline \multicolumn{4}{|l|}{ Safety (number of exacerbations/number of patients with indications during baseline) } \\
\hline Composite pain, $\mathrm{n}(\%)^{\mathrm{b}, \mathrm{c}}$ & $32 / 342$ & $220 / 1,463$ & $<0.01$ \\
\hline General pain & $10 / 185$ & $89 / 779$ & 0.02 \\
\hline Headache & $2 / 45$ & $5 / 181 \quad(2.8)$ & 0.63 \\
\hline Ankylosing spondylitis & $(3.7)$ & $24 / 349$ & 0.29 \\
\hline Gout & $0 / 25$ & $8 / 101$ & 0.35 \\
\hline Osteoarthritis & $11 / 164$ & $77 / 705$ & 0.11 \\
\hline Rheumatoid arthritis & $1 / 13$ & $8 / 63 \quad(12.7)$ & 1.00 \\
\hline Joint pain & $10 / 227$ & $57 / 953 \quad(6.0)$ & 0.36 \\
\hline Muscle spasm & $1 / 17$ & $0 / 70$ & 0.20 \\
\hline \multicolumn{4}{|l|}{ Economics } \\
\hline Change in cost of NSAID prescriptions per member per month, median change (IQR)d & $-\$ 0.29(-2.37,-0.11)$ & $-\$ 0.23(-2.59,0.00)$ & 0.054 \\
\hline \multicolumn{4}{|l|}{$\begin{array}{l}\text { aComposite: ER visit or hospitalization with a primary diagnosis of GI bleed or AKI. } \\
{ }^{b} \text { Chi-square test. } \\
\text { cComposite: ER visit or hospital visit with a primary diagnosis of at least } 1 \text { of } 8 \text { pain diagnoses. } \\
\text { dT-test or Wilcoxon rank-sum tests. }\end{array}$} \\
\hline
\end{tabular}

\section{Effectiveness Outcomes}

The effectiveness outcomes were the rates of new-onset composite GI bleed events and composite AKI, defined as having an ER visit or hospitalization with a primary diagnosis of GI bleed or AKI, respectively. The unadjusted rate of patients who experienced GI bleed events was significantly lower in the deprescribed group than in the usual care group (3.9\% vs. 6.6\%, respectively, $P=0.04$ ) and was primarily driven by hospitalization $(2.6 \%$ vs. $5.1 \%$, respectively, $P=0.03)$. For the unadjusted rate of AKI, there was no significant difference between the deprescribed group and the usual care group (2.3\% vs. 3.9\%, respectively, $P=0.11$; Table 2 ).

In addition to the common independent variables used in the conditional logistic regression model (Table 3), for the risk of GI bleed, we also adjusted for previous hospitalized GI bleed events, whereas for risk of AKI, we also adjusted for renal disease. The adjusted odds ratio (OR) for the deprescribed group experiencing GI bleed events was 0.65 (95\% confidence interval $[\mathrm{CI}]=0.36-1.16, P=0.15)$ in comparison with the usual care group (Table 3). The adjusted OR for the deprescribed group experiencing AKI was $0.53(0.24-1.16, P=0.11)$ in comparison with the usual care group (Table 3).

\section{Safety Outcome}

The safety outcome assessed the rate of ER visits or hospitalization because of an exacerbation of existing pain. The proportion of patients with composite pain, defined as having an ED visit or hospitalization with a primary diagnosis of at least 1 of the 8 pain diagnoses, was lower in the deprescribed group versus the usual care group $(9.4 \%$ vs. $15.0 \%, P<0.01)$. Among the individual pain diagnosis, the proportion of patients with exacerbation of general pain was also significantly lower in the deprescribed group versus the usual care group (5.4\% vs. $11.4 \%, P=0.02$ ). There was no difference between the deprescribed and usual care groups for the other 7 pain diagnoses (Table 2).

The adjusted OR for the deprescribed group experiencing exacerbation of pain was $0.50(95 \% \mathrm{CI}=0.33-0.77, P<0.01)$ in comparison with the usual care group (Table 3). Similarly to the effectiveness outcomes, we also adjusted for the common independent variables and renal disease.

\section{Economic Outcome}

There was no significant difference in the median change in monthly NSAID drugs costs PMPM between the deprescribed group versus the usual care group $(-\$ 0.29[95 \%$ $\mathrm{CI}=-2.37,-0.11]$ vs. $-\$ 0.23[-2.59,0.00], P=0.054$; Table 2).

\section{Discussion}

While there have been studies that have evaluated pharmacistmanaged deprescribing programs for other classes of medications, to our knowledge this is the first study to investigate the 


\begin{tabular}{|c|c|c|c|}
\hline $\begin{array}{l}\text { Conditiona } \\
\text { Risk of Gl B } \\
\text { Exacerbatic }\end{array}$ & $\begin{array}{l}\text { Logistic Re } \\
\text { eed Events } \\
\text { of Pain }\end{array}$ & $\begin{array}{l}\text { gression } \\
\mathrm{AKI} \text {, an }\end{array}$ & \\
\hline Variables & Odds Ratio & $95 \% \mathrm{CI}$ & $P$ Value \\
\hline \multicolumn{4}{|l|}{ Risk of GI bleed events ${ }^{a}$} \\
\hline Deprescribed vs. usual care group & 0.65 & $0.36-1.16$ & 0.15 \\
\hline \multicolumn{4}{|l|}{ Risk of $A K I^{b}$} \\
\hline Deprescribed vs. usual care group & 0.53 & $0.24-1.16$ & 0.11 \\
\hline \multicolumn{4}{|l|}{ Risk of exacerbation of painc } \\
\hline Deprescribed vs. usual care group & 0.50 & $0.33-0.77$ & $<0.01$ \\
\hline \multicolumn{4}{|c|}{$\begin{array}{l}\text { avariables that were used in the regression model included age, female, CCI score, } \\
\text { previous hospitalized GI bleed events, Caucasian, NSAID classes, and the } 8 \text { pain } \\
\text { diagnoses. } \\
\text { bVariables that were used in the regression model included age, female, CCI score, } \\
\text { renal disease, Caucasian, NSAID classes, and the } 8 \text { pain diagnoses. } \\
\text { cVariables that were used in the regression model included age, female, CCI score, } \\
\text { Caucasian, NSAID classes, renal disease, and the } 8 \text { pain diagnoses. } \\
\text { AKI =acute kidney injury; CCI =Charlson Comorbidity Index; CI = confidence } \\
\text { interval; GI = gastrointestinal; NSAID=nonsteroidal anti-inflammatory drug. }\end{array}$} \\
\hline
\end{tabular}

effectiveness, safety, and economic outcomes of a deprescribing program for NSAIDs in geriatric patients. We found that a pharmacist-managed deprescribing program was associated with a nonsignificant trend towards reduced rates of GI bleed events and AKI between the deprescribed group and the usual care group during the 6-month follow-up. We met the threshold of 272 patients in the pharmacist-managed group and 1,088 patients in the usual care group required to achieve $80 \%$ power with a type I error of 5\% in order to detect a decrease from $11 \%$ to $5 \%$ in the proportion of patients who experience GI bleeds. However, the event rate for GI bleed was much lower at $6 \%$ in the overall study cohort. In addition, the observed differences between the 2 groups were only $2.7 \%$. The risk of GI events occurring from NSAID use is often dose-dependent and linearly related to continuous use over time. ${ }^{13}$ The small difference of GI bleed events in our study may be due to a short follow-up time. In addition, although the use of NSAIDs may pose a risk of renal dysfunction, elderly patients often exhibit other risk factors, such as older age, sepsis, surgical procedures, or comorbidities, all of which may cumulatively place them at risk for AKI. ${ }^{14}$ Therefore, adverse effects of GI bleed and AKI may be more detectable with a longer follow-up time.

There were no observed drug cost savings from deprescribing NSAIDs, since there was no significant differences in the median change in cost of NSAID prescriptions PMPM between the 2 groups. These results may be a result of a number of factors, such as changes in behavioral patterns of patients in both groups taking NSAIDs that range from continuous use to as needed basis or decreased doses of NSAID medications versus discontinuation of the drug. Although we did not observe a medication cost benefit for the study group, the cost benefit from a lower rate of pain exacerbation-a composite endpoint of ER visits or hospitalization with a primary diagnosis of a pain diagnosis-may be attributed through decreased acute care use and generalized in future cost benefit analyses.

There were several strengths of this study. The study design allowed us to compare the deprescribed and usual care groups using propensity scores to match both groups for various covariables and reduce selection bias. Furthermore, this study was conducted in an integrated health care system, allowing access to a robust EMR such that patient health information and data are readily accessible to perform outcomes analyses. Finally, the deprescribing program's pharmacists were able to provide consistent interventions using a well-structured protocol that is maintained by a partnership between physicians and pharmacists.

\section{Limitations}

This study has a number of limitations. Some limitations were associated with a retrospective study design, small sample size, and a short follow-up period. There may be unobservable variables that accounted for differences between patients in the usual care group and the deprescribing group. For instance, factors such as lifestyle habits, diet, and over-thecounter NSAID or aspirin use may have affected the outcomes; however, this data could not be extracted. Since the study used observable demographic variables and characteristics to select for a control group using a propensity score study design, we were unable to account for unobservable variables.

We also did not collect information on other related outcomes, such as frequency of physician visits, and covariables, such as other medications used and the "intensity" of NSAID used at baseline that may affect outcomes. However, this study has provided key insights on the effect of deprescribing NSAIDs in geriatric patients, and the researchers of this study hope to continue sharing the results and best practices with various stakeholders rather than wait for a larger sample or a longer follow-up time.

Pain-related outcomes in studies are commonly measured using patient-reported pain scores in literature ${ }^{15}$; however, our study did not conduct chart reviews to collect patientreported pain scores or the severity of pain-related outcomes. We used ICD-9-CM and ICD-10-CM codes to define these pain diagnoses. Our results showed a 2-fold decrease in rate of exacerbation of pain in the pharmacist-managed deprescribed group versus the usual care group. It may be possible that the deprescribed group had less severe pain at baseline in comparison to the usual care group, but this cannot be differentiated by ICD codes. Patients in the deprescribed group may have developed a better understanding about their pain and/ or have used nonpharmacological options (e.g., acupuncture, ice/heat therapy, and turmeric or other supplements) after conversing with the deprescribing pharmacist. 


\section{Evaluation of a Pharmacist-Managed Nonsteroidal Anti-Inflammatory Drugs Deprescribing Program in an Integrated Health Care System}

Finally, because the patients in the study were from one geographic area in a closed health care system, it may be difficult to generalize the results outside of an integrated health care system.

\section{Conclusions}

Overall, a pharmacist-managed NSAID deprescribing program was implemented to reduce chronic NSAID use in elderly patients with risk factors for adverse events. The outcomes analysis showed a nonsignificant trend towards reduced rates of GI bleed events and AKI between the deprescribed group and the usual care group. There was, however, a statistically significant 2-fold decrease in the rate of exacerbation of pain in the pharmacist-managed deprescribed group versus the usual care group. Finally, no differences in the median change in monthly NSAID drug costs PMPM were observed. Future directions of this study may involve conducting a prospective study with a longer follow-up period to validate the findings at KPNC; including a larger cohort in order to study differences among NSAID classes; including other economic outcomes, such as health care resources use, and other effectiveness outcomes such as cardiovascular events; and exploring deprescribing of NSAIDs in other clinical settings.

\section{Authors}

REZOANA RASHID, PharmD, BCPS, Ambulatory Care Pharmacy, Kaiser Permanente Los Angeles Medical Center, Los Angeles, California. CHRISTOPHER CHANG, PharmD, BCGP, PMP, and LYNN DEGUZMAN, PharmD, BCGP, Clinical Pharmacy Services, Kaiser Permanente Northern California Region, Oakland. FANG NIU, MS, Pharmacy Outcomes Research Group, Kaiser Permanente California Regions, Downey. MAISHA DRAVES, MD, MPH, and SAMEER AWSARE, MD, FACP, The Permanente Medical Group, Kaiser Permanente Northern California Region, Oakland. RITA HUI, PharmD, MS, Pharmacy Outcomes Research Group, Kaiser Permanente California Regions, Oakland.

AUTHOR CORRESPONDENCE: Rita Hui, PharmD, MS, Pharmacy Outcomes Research Group, Kaiser Permanente California Regions, 1800 Harrison St., \#1301, Oakland, CA 94612. Tel.: 510.625.3948; E-mail: Rita.L.Hui@kp.org.

\section{DISCLOSURES}

No funding was provided to support this research study. The authors of this study have no actual or potential conflicts of interest to report.

Parts of this study were presented in a nonreviewed resident poster at the AMCP Managed Care and Specialty Pharmacy Annual Meeting; March 25-28, 2019; San Diego, CA.

\section{ACKNOWLEDGMENTS}

The authors thank Doris J. Kao, PharmD, BCPS, FCSHP; Anne Tran-Pugh, PharmD, FCSHP; Gary Besinque, PharmD, FCSHP; and Denise Harano, PharmD, BCPS, BCGP, for their support.

\section{REFERENCES}

1. Ortman JM, Velkoff VA, Hogan H. An aging nation: the older population in the United States: population estimates and projections. Current Population Reports. Report number: P25-1140. May 2014. Available at: https://www.census.gov/library/publications/2014/demo/p25-1140.html. Accessed May 15, 2020

2. Masnoon N, Shakib S, Kalisch-Ellett L, Caughey GE. What is polypharmacy? A systematic review of definitions. BMC Geriatr. 2017;17(1):230.

3. Garfinkel D, Ilhan B, Bahat G. Routine deprescribing of chronic medications to combat polypharmacy. Ther Adv Drug Saf. 2015;6(6):212-33.

4. Page AT, Clifford RM, Potter K, et al. The feasibility and effect of deprescribing in older adults on mortality and health: a systematic review and meta-analysis. Br J Clin Pharmacol. 2016;82(3):583-623

5. Hui RL, Chang CC, Niu F, et al. Evaluation of a pharmacist-managed antidiabetic deprescribing program in an integrated health care system. J Manag Care Spec Pharm. 2019;25(8):927-34. Available at: https://www.jmcp.org/ doi/10.18553/jmcp.2019.25.8.927.

6. Farrell B, Pottie K, Rojas-Fernandez CH, et al. Methodology for developing deprescribing guidelines: using evidence and GRADE to guide recommendations for deprescribing. PLoS One. 2016;11(8):e0161248.

7. Steinman MA. Polypharmacy-time to get beyond numbers. JAMA Intern Med. 2016;176(4):482-83.

8. Wongrakpanich S, Wongrakpanich A, Melhado K, Rangaswami J. A comprehensive review of non-steroidal anti-inflammatory drug use in the elderly. Aging Dis. 2018;9(1):143-50.

9. Von Soest EM, Valkhoff VE, Mazzaglia G, et al. Suboptimal gastroprotective coverage of NSAID use and the risk of upper gastrointestinal bleeding and ulcers: an observational study using three European databases. Gut. 2011;60(12):1650-59

10. Pilotto A, Franceschi M, Leandro G, Di Mario F. NSAID and aspirin use by the elderly in general practice: effect on gastrointestinal symptoms and therapies. Drugs Aging. 2003;20(9):701-10.

11. Martin P, Tamblyn R, Benedetti A, et al. Effect of a pharmacist-led educational intervention on inappropriate medication prescriptions in older adults: the D-PRESCRIBE randomized clinical trial. JAMA. 2018;320(18):1889-98.

12. Quan H, Sundararajan V, Halfon P, et al. Coding algorithms for defining comorbidities in ICD-9-CM and ICD-10 administrative data. Med Care. 2005;43(11):1130-39.

13. Goldstein JL, Cryer B. Gastrointestinal injury associated with NSAID use: a case study and review of risk factors and preventative strategies. Drug Healthc Patient Saf. 2015;7:31-41.

14. Chao CT, Tsai HB, Wu CY, et al. Cumulative cardiovascular polypharmacy is associated with the risk of acute kidney injury in elderly patients. Medicine (Baltimore). 2015;94(31):e1251.

15. Scascighini, L, Toma V, Dober-Spielmann S, et al. Multidisciplinary treatment for chronic pain: a systematic review of interventions and outcomes. Rheumatology. 2008;47(5):670-78. 


\section{Evaluation of a Pharmacist-Managed Nonsteroidal Anti-Inflammatory Drugs Deprescribing Program in an Integrated Health Care System}

\section{APPENDIX}

ICD-9-CM, ICD-10-CM, and DRG Codes Used in this Study

Gastrointestinal bleed or disorders (ulcer and severe esophagitis)

ICD-9-CM: 251.5, 530, 531, 532, 533, 534, 535, 536.8, 537.84, 562.12, 562.13

$569.3,569.84,569.85,578$

ICD-10-CM: K20, K22.1, K22.7, K25, K26, K27, K28, K92.1, K92.2, N17, Z87.11 DRG: $377-384,388-392$

Cardiovascular disease (myocardial infarction, stroke, transient ICD-9-CM: 410, 412, 414, 428, 429.20, 433, 434.91, 437

ischemic attack, atherosclerotic cardiovascular disease, or heart failure)

ICD-10-CM: G45.8, G45.9, I21, I22, I23, I25, I50, I63, Z86.73

End-stage renal disease

ICD-9-CM: 585.6

ICD-10-CM: I12, N18.6

DRG: 682-685

ICD-9-CM: 584

ICD-10-CM: N17

Acute kidney injury

DRG: $682-685$

General pain

ICD-9-CM: 250.6, 338, 350, 356, 357, 725, 728.81, 729.1, 780.96, 789, 847

ICD-10-CM: A52.15, B02.23, E08.40, E09.40, E10.40, E11.40, E12.40, E13.40, G13.0, G64, G65, G89, M34.83, M54.2, M60, M79.1, M79.8, M79.9, R10, R52

Headache

ICD-9-CM: 307.81, 339, 346, 350.2, 784.0

ICD-10-CM: G43, G44, G50.1, R51

Ankylosing spondylitis

ICD-9-CM: 720,721

ICD-10-CM: M24.6, M26.6, M45, M46, M47, M48, M49

ICD-9-CM: 274

ICD-10-CM: M1A, M10

Gout

ICD-9-CM: 715

Osteoarthritis

ICD-10-CM: M15-M19

ICD-9-CM: 714, 725

ICD-10-CM: M05, M06

Rheumatoid arthritis

ICD-9-CM: 719.4, 724.1, 724.2, 724.5, 722, 723.1

Joint pain

ICD-10-CM: M25.5, M50, M54.2, M54.5, M54.6, M79.6, M79.64, M79.67

ICD-9-CM: 728.85

Muscle spasm

ICD-10-CM: M25.2, M62.4, M62.8

DRG=Diagnosis-Related Group; ICD-9/10-CM = International Classification of Diseases, Ninth/Tenth Revision, Clinical Modification. 